\title{
Una apología de las posturas funcionalistas del progreso científico
}

\author{
A defence of functionalist approach to scientific progress*
}

\author{
DAMIAN ISLAS MONDRAGÓN**
}

\begin{abstract}
Resumen: Analizo los principales argumentos en torno a la dependencia teórica de los enunciados y conceptos observacionales tomando como eje de discusión la postura de Gerhard Schurz. Después discuto los principales argumentos sobre la dependencia teórica de la percepción. Finalmente analizo la dependencia teórica de la experimentación científica teniendo como eje de discusión la postura de Allan Franklin. Muestro que un rasgo positivo de la dependencia teórica de la observación y la experimentación es que una teoría científica puede establecer los mecanismos para evaluar la importancia de los fenómenos observados inesperados. Estos mecanismos sin duda constituyen un instrumento esencial para el progreso científico.

Palabras clave: Dependencia teórica de la Observación. Dependencia Teórica de la Percepción. Dependencia Teórica de la Experimentación. Conceptos teóricamente neutrales. Progreso Científico.
\end{abstract}

\begin{abstract}
I analyze the main arguments about theory-dependence of scientific observational sentences and concepts. I discuss the proposal of Gerhard Schurz on the matter. Then I discuss the main arguments about theory-dependence of human perception. Finally, I analyze theorydependence of scientific experimentation. I discuss the proposal of Allan Franklin on the matter. I show that a positive feature of theoretical dependence of observation and experimentation is that a scientific theory could establish the necessary methodological mechanisms with which scientists might recognizing and evaluating the cognitive importance of observed unexpected phenomena. These mechanisms undoubtedly constitute an essential tool for scientific progress. Keywords: Theory-Dependence of Observation. Theory-Dependence of Perception. TheoryDependence of Experimentation. Theory-Neutral Observation Concepts. Scientific Progress.
\end{abstract}

Recibido: 07/08/2014. Aceptado: 19/02/2015.

* Este artículo fue realizado con el apoyo del Consejo Nacional de Ciencia y Tecnología (CONACYT) de México para realizar una estancia sabática de investigación en el Centro Peninsular en Humanidades y en Ciencias Sociales (CEPHCIS) de la UNAM.

** Orcid: 0000-0001-8538-6835. Docente e Investigador del Instituto de Ciencias Sociales de la Universidad Juárez del Estado de Durango. Líneas de investigación: Filosofía de la Ciencia. Progreso Cognitivo de la Ciencia. Experimentos Mentales. Últimas publicaciones: Teorías contemporáneas del progreso científico: un análisis filosófico en torno al progreso cognitivo de la ciencia, Plaza y Valdés, México, 2015; "La falsación empírica y los problemas lacunae". Revista de Filosofía, Vol. LIII, No. 137, 2014, pp. 33-41. Universidad de Costa Rica.Contacto: damianislas@ujed.mx / damian.islasmondragon@utoronto.ca 


\section{Introducción}

El lenguaje científico que utiliza la ciencia suele ser dividido en dos tipos, a saber, el lenguaje observacional -con el cual son designadas las relaciones y propiedades de entidades y procesos científicos observados (y observables) - y el lenguaje teórico con el cual los científicos se refieren a las entidades y procesos científicos inobservados (e inobservables). Cada vez que un nuevo instrumento científico es inventado, se cree que la probabilidad de que un enunciado o concepto teórico se convierta en un concepto observacional se incrementa, motivando el progreso cognitivo de la ciencia ${ }^{1}$.

No obstante, se ha argumentado que los enunciados observacionales exhiben una fuerte dependencia teórica al grado de convertir a la ciencia empírica en una práctica epistémica "circular" cuya producción de conocimiento objetivo puede ponerse seriamente en duda. De ser así, convertir enunciados teóricos en enunciados observacionales vía el perfeccionamiento tecnológico no sería una condición suficiente para el progreso de la ciencia.

Para desarrollar mi estudio, primero analizo críticamente los principales argumentos en torno a la dependencia teórica de los enunciados y conceptos observacionales tomando como eje de discusión la propuesta de Gerhard Schurz (2015) sobre los conceptos observacionales teóricamente neutrales. Después discuto algunos de los principales argumentos con los que se defiende la dependencia teórica de la percepción. Posteriormente reviso algunos de los argumentos que sostienen la dependencia teórica de la experimentación científica teniendo como eje de discusión la propuesta de Allan Franklin (2015). A lo largo del texto muestro que lo que es potencialmente observable, así como lo que es potencialmente no observable desde un punto de vista cognitivo parece depender de las teorías que los científicos asumen previamente y con las cuales configuran sus expectativas y formas de ver el mundo. No obstante, esta dependencia teórica no es del todo negativa y cumple una función importante para el progreso científico ya que puede servir para establecer los mecanismos metodológicos necesarios con los que puede ser detectada y evaluada la importancia cognitiva de los fenómenos observados inesperadamente. Finalmente, presentaré algunas conclusiones que pueden inferirse del presente estudio.

\section{La dependencia teórica de los conceptos observacionales}

Las teorías filosóficas que afirman la fuerte dependencia teórica de los enunciados observacionales sostienen que la ciencia empírica es una práctica epistémica "circular" incapaz de producir conocimiento objetivo del mundo. Veamos por qué. Supóngase que $\mathrm{T}_{1}$ y $\mathrm{T}_{2}$ son dos teorías científicas propuestas para explicar y predecir ciertos fenómenos y procesos observables y que ambas teorías pueden estar sujetas a cierto grado de corroboración empírica. Debido a que la evidencia científica en pro o en contra de ambas teorías consistiría en confirmar o en refutar algunas de sus predicciones observacionales, si la evidencia empírica

1 Podemos dividir a los instrumentos científicos en tres tipos, a saber, (I) aquellos que extienden el dominio de lo que puede ser observable como los microscopios, los telescopios, los amplificadores, etc.; (II) aquellos que permiten establecer regularidades como el barómetro o el termómetro y (III) aquellos que permiten inferir la existencia de alguna entidad física inobservable en $\mathrm{t}_{1}$ como la cámara de gas de Wilson, el microscopio electrónico o los tomógrafos por emisión de positrones o por imágenes de resonancia magnética. 
fuera totalmente dependiente de $\mathrm{T}_{1} \mathrm{o} \mathrm{T}_{2}$, respectivamente, entonces no existiría evidencia empírica independiente con la cual fuera posible refutar o confirmar las explicaciones y predicciones de estas teorías. Este caso hipotético convertiría en auto-justificables cada una de las explicaciones y predicciones emitidas tanto por $\mathrm{T}_{1}$ como por $\mathrm{T}_{2}$.

Por otro lado, si las explicaciones y predicciones empíricas que cada una de estas teorías emitiera fueran opuestas o suficientemente distintas entre sí, esto es, si ambas teorías fueran mutuamente inconmensurables, tendríamos que postular un tipo de evidencia neutral con la cual fuera posible evaluar de manera objetiva ambas teorías. Ciertamente, la postulación de una evidencia neutral de este tipo tendría que ser ella misma independiente no sólo de $\mathrm{T}_{1} \mathrm{y}$ $\mathrm{T}_{2}$, sino de cualquier otra teoría que inclinara la balanza a favor de alguna de las teorías bajo evaluación. En cualquier caso, tanto la auto-justificación de $\mathrm{T}_{1}$ y $\mathrm{T}_{2}$ como la postulación de una evidencia neutral con estas características, parece representar un reto para la formulación de una teoría acabada del progreso empírico de la ciencia.

Se ha $\operatorname{argumentado}^{2}$ que una posible solución a este dilema es hacer una distinción entre los conceptos que dependen de una teoría, digamos de $\mathrm{T}_{1}$, y los conceptos que dependen de teorías o hipótesis secundarias y auxiliares de $\mathrm{T}_{1}$. El primer tipo de conceptos es el que estaría directamente bajo prueba, mientras que los conceptos del segundo tipo lo estarían sólo de manera indirecta. Sin embargo, aunque con esta estrategia podemos evitar justificar ciertos conceptos ligados a teorías e hipótesis secundarias y auxiliares; todavía tenemos el problema de tener que justificar los conceptos ligados a la teoría principal. Como podemos esperar, tampoco es fácil alcanzar un consenso en relación a la construcción de una distinción clara y precisa entre estos dos tipos de conceptos, sobre todo cuando estamos evaluando empíricamente una teoría científica como un todo.

Por otro lado, dejar de evaluar -o hacerlo deficientemente- uno o más de los conceptos pertenecientes al ámbito de las teorías secundarias y auxiliares podría repercutir en la evaluación de los conceptos dependientes directamente de $T_{1}$. Como ha señalado correctamente Gerhard Schurz, al final de la cadena de conceptos bajo evaluación siempre quedarían algunos conceptos ligados a teorías empíricamente injustificadas lo que nos conduciría a una regresión justificatoria ad infinitum. De acuerdo con Schurz, para evitar esta regresión es necesario postular la existencia de conceptos observacionales teóricamente neutrales (Schurz 2015: 140).

Los conceptos observacionales teóricamente neutrales surgirían de la experimentación científica, por lo que no dependerían del conocimiento previamente aceptado por las teorías. De acuerdo con Schurz, para mostrar que las ciencias empíricas pueden alcanzar conocimiento objetivo, esto es, no circular, no auto-justificatorio ni regresivo; basta con refutar la tesis de la total dependencia teórica de los conceptos observacionales mediante la justificación de este tipo de conceptos teóricamente neutrales. A continuación analizaré la propuesta de Schurz con el objetivo de evaluar sus resultados y utilidad para solucionar el problema de la dependencia teórica -total o parcial- de los conceptos observacionales que emite la ciencia y su repercusión en la idea de progreso científico.

2 Véase Hempel, 1988: 154. 
Para comenzar nuestro análisis, lo primero que debemos precisar es el tipo de enunciados y conceptos observacionales que están bajo discusión. Un ejemplo aparentemente inocuo de un enunciado observacional es el siguiente: "este objeto es verde". Se ha argumentado que este tipo de enunciados trasciende el contenido de la experiencia subjetiva al ser expresados en un modo realista a través de enunciados como "este objeto". No obstante, es un hecho que nuestras creencias sobre estos objetos podrían ser erróneas y ciertamente el origen del error podría ser multifactorial, a saber, la alucinación, la impericia visual, etc.

De acuerdo con Schurz, aunque el argumento de que podríamos estar en un error al respecto de nuestras creencias sobre estos y otros objetos es correcto; esta situación no es atinente para el debate sobre la dependencia teórica de la observación el cual tiene que ver con el contenido de una obervación y su supuesta dependencia con el contenido de las teorías que asumimos previamente y que configuran nuestras expectativas y formas de ver el mundo. Que alguien asuma una postura epistemológica específica, por ejemplo una postura realista o una postura idealista de tipo fenomenológico, al respecto de la existencia o no de ciertos objetos en el mundo no afecta, afirma Schurz, el contenido de sus observaciones; sino sólo su interpretación. Como sabemos, el término "objeto" para un realista se refirere a un tipo de entidad que se asume está fuera de nuestra experiencia; mientras que para un fenomenalista dicha entidad se asume como interna a nuestra experiencia. No obstante, asumir cualquiera de estas posturas no cambiaría, sostiene Schurz, la observación de algo "verde" en una observación de algo "azul" (Schurz, 2015: 141).

Si bien Schurz acepta que las teorías que asumimos previamente determinan la selección de los problemas relevantes para la investigación científica y el tipo de observaciones que los científicos desean encontrar, éstas no determinan, sostiene este autor, el "contenido" de estas observaciones, esto es, los defensores de teorías incompatibles no necesariamente observan cosas diferentes al acercarse, por ejemplo, a un mismo experimento o a una misma área espacio-temporal específica. A lo más, las diferentes expectativas y formas de ver el mundo implícitas en cada teoría puede provocar que los científicos ignoren ciertas observaciones por considerarlas irrelevantes y se centren en otras que consideren importantes. De acuerdo con Schurz, una prueba inter-subjetiva -o en este caso inter-teórica- que evaluara de manera objetiva el contenido de las observaciones no sólo sería posible; sino que representaría una manera de evitar el dogmatismo científico (Schurz, 2015: 142).

Sin embargo, las cosas no parecen ser tan sencillas como piensa Schurz. Imaginemos nuevamente que dos teorías científicas -digamos $\mathrm{T}_{1} \mathrm{y}_{2}$ - tienen intereses comunes de investigación, pero exhiben incompatibles expectativas y formas de ver el mundo. Ciertamente $\mathrm{T}_{2}$ podría hacer notar un rasgo particular, digamos el rasgo $\mathrm{X}$, que $\mathrm{T}_{1}$ pasa por alto -de manera voluntaria o involuntaria- siempre y cuando X esté en el espectro de las observaciones que $\mathrm{T}_{2}$ no ignora. En el caso de la prueba intersubjetiva a la que se refiere Schurz entre dos teorías científicas distintas o entre dos tradiciones de investigación incompatibles, el rasgo X ignorado por una tradición $\operatorname{Tr}_{1}$ podría ser "traído a la conciencia" de los defensores de otra tradición $\operatorname{Tr}_{2}$ siempre y cuando $\mathrm{X}$ esté en el espectro de variables que la prueba analiza. Lo anterior implica, al menos, que lo que es potencialmente observable sí depende de las teorías y tradiciones de investigación, específicamente de su espectro o rango de visión.

Ahora bien, podríamos preguntarnos que pasaría si todas las teorías o tradiciones involucradas en la evaluación inter-teórica ignoran un rasgo $\mathrm{Y}$ específico, esto es, si ninguna 
tuviera la capacidad de reconocer Y debido al rango de visión implícito en todas estas teorías. La respuesta es que Y simplemente pasaría inadvertido para ciencia actual, lo que muestra de manera negativa que lo que es potencialmente no-observable para la ciencia también parece depender de las teorías o tradiciones científicas incluso cuando pensamos en éstas como un conjunto de teorías. Se argumentará que el debate sobre la dependencia teórica de la observación tiene que ver con rasgos observados y no con rasgos potencialmente observables o no-observables. Sin embargo, notemos a este respecto que aunque $\mathrm{T}_{2} \mathrm{O} \operatorname{Tr}_{2}$ pudieran hacer que $\mathrm{T}_{1}$ o $\operatorname{Tr}_{1}$ dirijan su atención a un rasgo $\mathrm{X}$ o $\mathrm{Y}$ que $\mathrm{T}_{1} \mathrm{o} \operatorname{Tr}_{1}$ no pueden ver a pesar de constituir rasgos pertinentes para sus ámbitos de investigación; esta situación implica, al menos, la dependencia de $\mathrm{T}_{1} \mathrm{O} \mathrm{Tr}_{1}$ de otras teorías y tradiciones para alcanzar cierto grado de objetividad explicativa y evitar lagunas explicativas.

Regresando al contenido cognitivo de los enunciados y conceptos observacionales, podemos clasificar las observaciones científicas en dos tipos. El primer tipo corresponde a las observaciones indirectas que se realizan con instrumentos científicos avanzados para detectar, por ejemplo, galaxias, bacterias o electrones. El segundo tipo corresponde a las observaciones que se realizan de manera directa como las percepciones visuales de colores, formas, etc. De acuerdo con Schurz, los datos científicos obtenidos a través de observaciones indirectas constituyen interpretaciones de observaciones realizadas de manera directa. Como tales, las observaciones indirectas son dependientes de las teorías aceptadas previamente con las cuales se justifica, por ejemplo, el funcionamiento correcto de un microscopio (como sabemos, el funcionamiento correcto de un microscopio involucra teorías mecánicas, ópticas, etc.) o la precisión de las mediciones que hace un telescopio (teorías matemáticas involucradas en su construcción). Sin embargo, los científicos son capaces de tener observaciones directas, afirma Schurz (2015: 147). Por ejemplo, cuando un químico dice haber "observado" un ácido basado en una prueba con papel tornasol, a lo que en realidad se refiere es que ha observado que el papel tornasol se tornó rojo. La inferencia que el científico hace en relación a la presencia de ácidos químicos a partir de este cambio de coloración es, en efecto, resultado de una interpretación teórica basada en una observación directa.

A este respecto, es sólo a partir de la existencia de cierta controversia en relación a lo que dos o más científicos infieren o interpretan de una observación directa, que la ciencia puede progresar cognitivamente al iniciarse cierta reflexión en torno a las asunciones teóricas implícitas que condujeron a los científicos a inferir diferentes resultados cognitivos. ${ }^{3}$ De acuerdo con Schurz, en este tipo de controversias las observaciones directas pueden servir como posibles generadores de acuerdos teóricos neutrales a los cuales los científicos pueden recurrir en caso de desacuerdos serios en sus interpretaciones.

Me parece que el argumento de Schurz presenta, al menos, dos problemas. Por un lado, no podemos negar que existe cierta dependencia teórica incluso en las observaciones directas. Cuando el químico "observó" que el papel tornasol se tornó "rojo”, este enunciado científico sólo pudo ser emitido involucrando una teoría, aunque sea mínima, del color ${ }^{4}$. Por otro lado, y aún más importante, es que Schurz no reconoce que existe cierta dependencia teórica que guía el proceso experimental que realiza el científico. Esto es, sin una teoría

3 Los desacuerdos en ciertos diagnósticos médicos son ejemplos de este tipo de controversias.

4 La misma crítica puede hacerse para el enunciado "este objeto es verde" que mencionamos más arriba. 
científica que le indique al científico qué hacer, ningún experimento se llevaría al cabo y, por lo tanto, la presencia del color rojo en el papel tornasol no tendría sentido. Más adelante profundizaré en la importancia que tiene la dependencia teórica de la experimentación científica para este debate.

Algo parecido sucede con el principio del relativismo lingüístico el cual sostiene que sólo podemos percibir lo que es describible conceptualmente. De acuerdo con Schurz, aunque podemos admitir que diferentes culturas han desarrollado diferentes sistemas conceptuales a partir de los cuales describen su propias percepciones, esto no implica que la experiencia perceptual en sí misma sea dependiente del lenguaje. Si los miembros de estas culturas no fueran capaces de aprender el significado de ciertos conceptos observacionales a través de un entrenamiento visual directo, esto es, vía ciertos actos demostrativos como "esto es verde" y "esto es azul", entonces el argumento de la dependencia lingüística de la experiencia sería válido, lo que rechaza Schurz (2015: 148).

En el fondo del argumento de Schurz existe el presupuesto de que es posible distinguir tajantemente entre la experiencia perceptual de un objeto y el lenguaje con el que concebimos dicha experiencia. A mí me parece que no es tan claro que sea posible distinguir con precisión entre el contenido "puro" de una observación y la descripción lingüística de dicho contenido, sobre todo si tomamos en cuenta que la manera de acceder a la información cognitiva del contenido de una observación es precisamente a través de nuestro lenguaje que le da "forma" al contenido de nuestras experiencias. Seguramente Schurz argumentaría que la "forma" y el "contenido" de una experiencia perceptual son dos cosas distintas. Sin embargo, lo que quiero enfatizar es que sin una forma adecuada, ningún contenido experiencial podría siquiera ser concebido. En otras palabras, el contenido de una experiencia conceptual es inextricable de la forma -en este caso lingüística- con la que traemos a nuestra conciencia cognitiva dicho contenido experiencial de la percepción.

Con los elementos hasta aquí analizados, Schurz estableció los elementos que considera deben poseer los conceptos observacionales teóricamente neutrales, a saber, (A) el criterio que define a un concepto observacional no es de tipo lógico; sino de tipo empírico y psicológico en el sentido de que es relativo a la capacidad sensorial humana; (B) un concepto observacional es un concepto cuya denotación puede ser reconocida a través de la percepción y $(\mathrm{C})$ un concepto observacional es aprendido vía experimentos no verbalizados del tipo "esto es verde" y "esto es azul" que involucra actos empíricamente corroborables que evitan los reportes introspectivos que pudieran confundir la percepción con interpretaciones teóricamente dependientes. Así, de acuerdo con Schurz, un concepto observacional es teóricamente neutral si y sólo si todos los humanos de diferentes culturas pueden comprenderlo a través de experimentos de aprendizaje demostrativo bajo condiciones físicas, biológicas y psicológicas normales de observación. Este aprendizaje es independientemente de la información, lenguaje y cultura que inevitablemente poseen las personas (Schurz, 2015: 150 y 151).

Como podemos ver, la tesis que defiende Schurz está fundamentalmente basada en el éxito que tengan los experimentos en torno al aprendizaje demostrativo con personas de diferentes culturas. Por supuesto, los resultado de este tipo de experimentos varía si se trata del aprendizaje de conceptos observacionales simples o complejos. Pero lo que quiero enfatizar aquí es que Schurz no toma en cuenta que los experimentos mismos pueden involucrar cierto 
grado de dependencia teórica en su formulación y realización. No obstante, antes de pasar al análisis de la dependencia teórica de la experimentación, conviene abordar el tema de la percepción dado que en el fondo del debate sobre la dependencia teórica de la observación existe un debate todavía más profundo en torno a dependencia teórica de la percepción que parece estar en la base de las observaciones científicas.

\section{La dependencia teórica de la percepción}

A este respecto, es famoso el ejemplo de la imagen del pato-conejo utilizado por Joseph Jastrow (1901: 295) la cual puede verse indistintamente como un pato o como un conejo, lo que evidenciaría, según algunos autores, la dependencia teórica de la percepción humana. ${ }^{5}$ De acuerdo con Schurz, el carácter constructivo de los procesos visuales sólo refutan el llamado Realismo Directo que sostiene que podemos ver las cosas de manera directa como son en sí mismas, esto es, sin la mediación de procesos - de carácter visual y neurológico- que construyen imágenes intermedias entre nosotros y los objetos que percibimos.

Ciertamente son pocos los autores que defienden el realismo directo, pero lo que enfatiza Schurz es que nuestra percepción y sus resultados son independientes de la información previamente aprendida. Muestra de ello es que a pesar de la advertencia que se le puede hacer a cualquier persona en relación a la ilusión visual que causan ciertas imágenes como la del pato-conejo, "todos" - afirma Schurz- tienden a seguir teniendo la misma ilusión perceptual. ${ }^{6}$ De acuerdo con Schurz, esto se debe a que el proceso humano de la percepción en general y de la visión en particular están basados en mecanismos innatos que hemos desarrollado durante millones de años de evolución, lo que explica por qué bajo condiciones "normales" -físicas, biológicas y psicológicas- podemos acceder a representaciones visuales correctas de la realidad; mientras que bajo ciertas circunstancias "anormales" sufrimos ilusiones ópticas. Por lo anterior, afirma Schurz, la dependencia de la percepción no ocurre en relación a la "información aprendida" previamente; sino, a lo más, en relación a las "teorías innatas" que todos los humanos comparten (Schurz, 2015: 144 y 145).

Analicemos el argumento de Schurz. Lo primero que hay que decir es que la dependencia de la percepción no ocurre en relación a las "teorías innatas" como afirma; sino en relación a los mecanismos innatos que supuestamente compartimos. Pero lo que me interesa enfatizar aquí es que la apelación a mecanismos "innatos" por parte de Schurz para explicar las ilusiones perceptuales y echar abajo el argumento de la dependencia teórica de la percepción asociado a las imágenes del tipo pato-conejo, parece no abordar la discusión actual en torno a la validez de las teorías innatistas en psicología y en las ciencias cognitivas.

5 En la misma línea argumentativa, Hanson ([1958] 1977: 13) reproduce una imagen que parece un antílope mientras que Wittgenstein ([1945] 2003: 447) reproduce la imagen original de Jastrow.

6 El tipo de imágenes como la del pago-conejo que pueden conducirnos a errores perceptuales son de tres tipos, a saber, (a) pobreza de estímulo (como en el caso de imágenes vistas en la oscuridad); (b) de alta complejidad (como en el caso de radiografías médicas) y (c) imágenes ambiguas. La imagen del pato-conejo pertenece a esta última clase. 
Como sabemos, uno de los principales problemas del innatismo o nativismo desde el punto de vista de las ciencias empíricas es que sus hipótesis carecen, por su propia naturaleza, de fundamentación empírica. No obstante, existen otros aspectos problemáticos todavía más fundamentales que están enfocados al concepto mismo de 'innato'. En este sentido, se argumenta que este concepto es un concepto "fundamentalmente confuso" al subsumir bajo un mismo término diferentes propiedades independientes entre sí. Algunas de las propiedades asociadas al concepto de "innato" son: (i) tener una explicación adaptativa evolutiva; (ii) ser insensible a la variación de factores extrínsecos; (iii) estar presente desde el nacimiento; (iv) ser universal en el sentido pan-cultural del término, esto es, estar presente en todas las culturas humanas y (v) no ser adquirido vía el aprendizaje (véase Griffiths, 1997; Bateson, 2000 y Oyama, 2000).

Una de las críticas que se le han hecho a los defensores de las teorías innatistas sobre todo en ciencias cognitivas es que confunden estas y otras propiedades bajo un mismo concepto: el de 'innato'. No obstante esta confusión, los defensores del innatismo realizan inferencias ilícitas al asegurar que si un rasgo evolutivo específico exhibe una de estas propiedades, es probable que dicho rasgo posea una o más de las otras propiedades (Griffiths, 1997: 60). Y ciertamente, al parecer Schurz también recurre a este tipo de inferencias ilícitas cuando de la propiedad (iv) infiere la propiedad (v), esto es, del argumento de que a pesar de las advertencias, todos tienden a seguir teniendo la misma ilusión perceptual al observar la imagen del pato-conejo, Schurz infiere sin justificación alguna que la dependencia de la percepción no ocurre en relación a la información aprendida. Notemos, finalmente, que Schurz había sostenido previamente que el contenido de las observaciones no depende de la postura epistemológica elegida. Sin embargo, al parecer Schurz comete la misma falta al desechar la idea de que la percepción no depende de la información adquirida aduciendo una postura biológica previa, esto es, el innatismo. En la siguiente sección analizaré algunos de los argumentos que se han formulado para mostrar la dependencia teórica de la experimentación. Dada la extensión del tema, en particular analizaré la postura al respecto desarrollada recientemente por Allan Franklin (2015).

\section{La dependencia teórica de la experimentación}

De acuerdo con la dependencia teórica de la experimentación, los conceptos observacionales teóricamente neutrales que defiende Schurz son imposibles si los conceptos con los que son descritos los resultados experimentales de la investigación científica poseen diferentes e incompatibles significados. El ejemplo utilizado por Franklin es el concepto de 'masa' el cual en la mecánica de Newton se refiere a una constante, mientras que en la mecánica relativista de Einstein este mismo concepto depende de la velocidad del objeto (Franklin, 2015: 157).

Franklin afirma que a pesar de que los defensores de ambas posturas teóricas competidoras describen cada procedimiento experimental de manera distinta, ambos pueden estar de acuerdo en que ciertamente las respectivas predicciones y mediciones que se desprenden de cada procedimiento son genuinamente obtenidas. Por lo anterior, Franklin considera que el verdadero problema de la inconmensurabilidad está en el nivel experimental y no en el nivel teórico (Franklin, 2015: 158). 
El primer problema con los procedimientos experimentales utilizados en ciencia es que éstos pueden depender de una teoría no sólo desde un punto de vista cognitivo, sino metodológico. Desde el punto de vista cognitivo, como consignamos más arriba, la emergencia de un rasgo observacional $\mathrm{Z}$-o de manera negativa, la no emergencia del rasgo $\mathrm{Z}$ - puede depender a tal grado de la formulación y alcance de los procedimientos experimentales que difícilmente podemos aceptar que $\mathrm{Z}$ sea una evidencia confiable de la metodología experimental sin caer en la circularidad argumentativa mencionada en la primera sección. Por lo anterior, la dependencia teórica de los procedimientos experimentales puede ser analizada en los mismos términos con los que hemos analizado la dependencia teórica de los conceptos observacionales.

Desde el punto de vista metodológico, los procedimientos experimentales pueden estar diseñados de tal manera que su ejecución excluya, de manera consciente, la observación de fenómenos no predichos por la teoría o tradición que está detrás de la formulación teórica del experimento con el fin de alcanzar el éxito empírico esperado. En esta misma línea argumentativa, también puede ocurrir que el efecto que tiene una observación no esperada sea minimizado voluntariamente con el mismo fin. Finalmente, puede darse el caso de que los procedimientos experimentales sean forzados o manipulados con el objetivo de explicar exitosamente un rasgo observacional acorde con una teoría previamente aceptada.

En todos estos casos, lo que me interesa enfatizar es que la emergencia -o no emergencia- de un rasgo específico $\mathrm{Z}$ en el procedimiento experimental causada por omisiones, fallas o manipulaciones conscientes o inconscientes de los datos observacionales obtenidos, puede repercutir en una deficiente interpretación de las observaciones empíricas y/o en la imposibilidad de observar un efecto observacional en particular. En todo caso, un rasgo positivo de la dependencia teórica de la observación y/o de los procedimientos experimentales es que la teoría puede establecer los mecanismos necesarios para reconocer y evaluar la importancia que los fenómenos observados no predichos por la teoría pueden tener para los objetivos cognitivos particulares que se buscan en un momento específico. Estos mecanismos sin duda constituyen un instrumento esencial para el progreso científico.

Por supuesto, no todos los datos teóricos se involucran en el diseño o en la construcción de los procedimientos experimentales. Sin embargo, es un hecho que existen lo que Franklin llama "cortes" que se aplican a los datos en sí mismos o a los análisis que se hacen de los resultados obtenidos experimentalmente. De acuerdo con Franklin, una manera de validar los resultados obtenidos experimentalmente es variar estos cortes y corroborar si los resultados son estables (Franklin, 2015: 162). No obstante, como es de esperarse, tales cortes también repercuten en la interpretación de los resultados obtenidos. Pero lo que me interesa enfatizar aquí, finalmente, es que la utilización de ciertas porciones de datos -lo que implica la exclusión de otras porciones- y la decisión de variar los cortes de una manera determinada y no de otra puede ser una manera de "camuflar" las teorías que se asumen previamente y que están involucradas en el diseño y construcción de los procedimientos experimentales.

Ciertamente, el involucramiento teórico en la construcción y desarrollo de los procedimientos experimentales "vicia" los resultados empíricos obtenidos, mermando la capacidad de la ciencia de producir conocimiento objetivo del mundo. Por lo anterior, la dependencia teórica de los procedimientos experimentales sigue siendo un reto importante para la construcción de una teoría coherente del progreso científico metodológico. 


\section{Conclusiones}

Hemos visto que el tema de la dependencia teórica no se reduce a los enunciados y conceptos utilizados en la ciencia ni a los procedimientos experimentales que realiza. En el fondo de esta discusión también está el tema de la dependencia teórica de la percepción humana. De acuerdo con Schurz, el proceso humano de la percepción está basado en mecanismos innatos desarrollados durante millones de años que explican por qué podemos obtener representaciones correctas e incorrectas. Por su parte, Franklin sostuvo que ante la inconmensurabilidad teórica, dos o más teorías en competencia pueden estar de acuerdo en que las respectivas predicciones y mediciones que se desprenden de cada procedimiento experimental son genuinamente obtenidas, lo que soluciona el problema de la dependencia teórica de los conceptos científicos. Para Franklin, el verdadero problema de la inconmensurabilidad teórica está en el nivel experimental.

A partir del análisis de los diferentes argumentos en pro y en contra de la dependencia teórica de los enunciados observacionales, de los límites de la percepción humana y de los diferentes procedimientos experimentales utilizados en la ciencia; argumenté que es útil trazar una distinción metodológica entre las relaciones y propiedades de entidades y procesos científicos observados/inobservados y observables/inobservables. Los primeros pertenecen al lenguaje teórico mientras que los segundos pertenecen al lenguaje observacional que utiliza la ciencia. ${ }^{7}$ Esta distinción nos permitió establecer que lo que es potencialmente observable y lo que es potencialmente no-observable sí depende de las teorías y tradiciones científicas.

En relación al debate sobre la dependencia teórica de la percepción mostré que las teorías innatistas son insuficientes para explicar por qué una experiencia conceptual es inextricable de la forma linguiística con la que traemos a nuestra conciencia cognitiva el contenido experiencial de la percepción. Finalmente, argumenté que la emergencia -o no emergenciade un rasgo específico en el procedimiento experimental causada por omisiones, fallas o manipulaciones conscientes o inconscientes de los datos observacionales obtenidos, puede repercutir en una malograda interpretación de las observaciones empíricas que sirven como evidencia científica.

Una clara consecuencia epistémica de esta situación es que, al parecer, ningún experimento crucial utilizado incluso como hipótesis auxiliar puede confirmar o refutar de manera conclusiva la pretensión que tiene cada teoría y tradición de investigación en competencia de ser un instrumento epistémicamente confiable para producir explicaciones y predicciones objetivas y racionales de los fenómenos y procesos que postulan. Para evadir esta consecuencia epistémica, todavía debemos indagar cómo podemos justificar los resultados cognitivos obtenidos a través de la experimentación científica sin caer en inferencias inválidas como la circularidad y auto-justificación argumentativa.

7 En otro lugar analizo más argumentos sobre la distinción entre el lenguaje teórico y el lenguaje observacional desde un puno de vista epistemológico (Islas, 2016). 


\section{Referencias bibliográficas}

Bateson, P. (2000), "Talking the Stink out of instinct" en H. Rose y S. Rose (eds.), Alas, Poor Darwin, London: Vintage.

Franklin, A. (2015), “The Theory-Ladennes of Experiment”, Journal for General Philosophy of Science, Vol. 46, No. 1, 2015, pp. 155-166.

Griffiths, P. (1997), What Emotions Really Are, Chicago: University of Chicago Press.

Hanson, N. (1977), Patterns of Discovery, Cambridge: Cambridge University Press, [1958].

Hempel, C. (1988), "A Problem concerning the Inferential Function of Scientific Theories", Erkenntnis, Vol. 28, No. 2, pp. 147-164.

Islas, D. (2016), "La distinción metodológica entre el lenguaje teórico y el lenguaje observacional: un análisis epistemológico", Andamios, Revista de Investigación Social, Vol. 31, mayo-septiembre, en prensa.

Jastrow, J. (1901), Fact and Fable in Psychology, London: MacMillan.

Oyama, S. (2000), Evolution's Eye. Durham: Duke University Press.

Schurz, G. (2015) "Ostensive Learnability as a Test Criterion for Theory-Neutral Observation Concepts", Journal for General Philosophy of Science, Vol. 46, No. 1, 2015, pp. 139-153.

Wittgenstein, L. (2003), Investigaciones Filosóficas, Ciudad de México: UNAM, [1958]. 
